\title{
Fächerunterschiede in der digitalen Hochschulbildung - eine Analyse auf Basis von Publikationsdaten
}

\section{Philipp Gross, Alexandra Shajek, Annette Stelter, Katia Tödt und Nicolas Winterhager}

\section{Zusammenfassung}

Der vorliegende Beitrag untersucht anhand eines Scoping Reviews, wie sich die Nutzung digitaler Formate, Methoden und technologischer Ansätze des Lehrens und Lernens an Hochschulen im Zeitverlauf (1971-2019) verändert hat. Neben allgemeinen Entwicklungstrends stehen dabei insbesondere fachspezifische Unterschiede im Fokus, da erste Forschungsarbeiten darauf hindeuten, dass die Digitalisierung der Lehre in den einzelnen Disziplinen mit unterschiedlichen Schwerpunkten erfolgt (zum Beispiel Dicheva et al. 2015; Hoyer und Mundt 2017; Kaeding und Scholz 2012). Datengrundlage der Analyse bilden Publikationen aus der Datenbank Scopus, die sich mit digitaler Hochschulbildung beschäftigen. Im Ergebnis zeigen sich punktuelle Unterschiede zwischen den Fächergruppen im wissenschaftlichen Diskurs zur digitalen Hochschulbildung. So sind beispielsweise Publikationen zu MOOCs besonders im Bereich der Mathematik und Naturwissenschaften zu finden.

\footnotetext{
P. Gross · A. Shajek $(\varangle) \cdot$ A. Stelter $\cdot$ K. Tödt $\cdot$ N. Winterhager Institut für Innovation und Technik (iit), Berlin, Deutschland E-Mail: shajek@iit-berlin.de

P. Gross

E-Mail: philipp.gross@iit-berlin.de
}

A. Stelter

E-Mail: stelter@iit-berlin.de

K. Tödt

E-Mail: toedt@iit-berlin.de

N. Winterhager

E-Mail: winterhager@iit-berlin.de 
Gamification ist besonders in den Ingenieurwissenschaften ein Thema und problem based learning im Bereich Humanmedizin/Gesundheitswissenschaften. Diese fachspezifischen Besonderheiten lassen sich jedoch in deutlich geringerem Ausmaß nachweisen als erwartet. Die Ergebnisse ließen sich so interpretieren, dass die Chancen der Digitalisierung, die sich insbesondere in der Verknüpfung mit den Besonderheiten der jeweiligen Fachkultur für die Hochschullehre ergeben, noch nicht genügend ergriffen werden.

\section{Schlüsselwörter}

Scoping Review • Digitale Lehr-Lernmethoden • Digitale Lehr-Lernformate • Bildungstechnologie $・$ Digitale Hochschulbildung

\section{$1 \quad$ Einleitung und theoretischer Hintergrund}

Die Digitalisierung umfasst alle Gesellschaftsbereiche und verändert auch die Hochschulen und die Lehre an Hochschulen (siehe zum Beispiel Schünemann und Budde 2018). Lehrende stehen vor der Herausforderung, Chancen der Digitalisierung zu nutzen und Benachteiligung oder Hürden für ihre Studierenden und ihre eigene Arbeit zu minimieren. Ansätze digitaler Lehr-Lernformate und -Methoden werden inzwischen an jeder Hochschule entwickelt oder sind bereits implementiert. Nicht zuletzt im Zuge der Coronaviruspandemie im Jahr 2020 hat das Thema an den Hochschulen einen erheblichen Bedeutungszuwachs erfahren, da Präsenzlehre zu einem großen Teil durch digitale Lehre ersetzt werden musste.

Innovative Ideen und Konzepte werden oft projekthaft an einzelnen Fachbereichen oder Fakultäten vorangebracht, häufig getragen von einzelnen engagierten Personen oder Teams. Es ist jedoch davon auszugehen, dass Fragen digitaler Lehre inzwischen flächendeckend an Hochschulen und in allen wissenschaftlichen Disziplinen und Fächern diskutiert werden. Welche (unterschiedlichen) digitalen Formate, Methoden und technologischen Ansätze dabei in den einzelnen Fächern besonders im Mittelpunkt stehen, ist bislang noch nicht hinreichend untersucht worden. Dabei ist diese Frage von zentraler Bedeutung für eine bestmögliche Nutzung der Chancen in einem jeweiligen Fach, die mit der Digitalisierung einhergehen. Im Hinblick auf die optimale Einbindung digitaler Lernumgebungen und -technologien sowie Lehr-Lernformate und -Methoden existieren zwar vielfältige Vorgehensweisen, die fächerübergreifend nutzbar sind. Gleichzeitig gibt es jedoch auch fachspezifische Besonderheiten und fachdidaktische Zugänge, die unterschiedliche digitale Formate oder Tools erfordern. 
So fordert etwa die Kulturministerkonferenz (KMK) in ihren Empfehlungen zur Digitalisierung der Hochschulen ,eine auf die Inhalte der einzelnen Fachdisziplinen bezogene, dem jeweiligen Thema angemessene Nutzung digitaler Medien“" (KMK 2019, S. 11) und entsprechende curriculare Verankerungen digital unterstützter Lehr-Lernformen. Um fachbezogene Lehrkonzepte für Lehrende zur Verfügung stellen zu können, bedarf es eines intensiven hochschulübergreifenden Austausches innerhalb der einzelnen Fachdisziplinen über die wirksame didaktische Einbindung digitaler Lehr-Lern-Materialien in die Hochschullehre (KMK 2019). Auch Mayrberger kommt nach einer Analyse fachspezifischer Besonderheiten beim Integrationsprozess von E-Learning in den Geistes-, Sozial- und Naturwissenschaften zu dem Schluss, dass ,die Integration von E-Learning-2.0Elementen eher dort didaktisch sinnvoll ist, wo kommunikative und kooperative Lehr- und Lernformen zur Fachkultur gehören“ (Mayrberger 2008, S. 166). Seit dieser ersten Analyse im Jahr 2008 hat sich die digitale Lehre an den Hochschulen stark weiterentwickelt und es ist interessant zu untersuchen, ob und inwiefern sich inzwischen die Nutzung verschiedener digitaler Lehr-Lernmethoden, -formate, -technologien oder -tools in verschiedenen Fächern unterschiedlich entwickelt hat.

Einen ersten Hinweis geben die aktuellen Praxisprojekte zur digitalen Hochschulbildung. Im vom Bundesministerium für Bildung und Forschung geförderten Forschungsprojekt Learnmap wurden Praxisprojekte untersucht und eine Projektdatenbank aufgebaut. ${ }^{1}$ Darin sind 136 Praxisprojekte an deutschen Hochschulen verzeichnet (Stand April 2020), die nach Fächern differenziert ausgewiesen werden. Dabei zeigt sich, dass die meisten der gelisteten Projekte in den Ingenieurwissenschaften (30), den Sozial- und Gesellschaftswissenschaften (19) und in der zusammengefassten Fächergruppe Mathematik und Naturwissenschaften angesiedelt sind (20), gefolgt von Lehramt (12), Medizin und Gesundheitswissenschaften (11) sowie Sprach- und Kulturwissenschaften (11). ${ }^{2}$ Die Projekte liefern einen ersten Einblick in die digitale Lehrpraxis in den Fächern. Für einen systematischen Fächervergleich sind diese Daten jedoch nicht ausreichend repräsentativ: Zum einen ist es von der Eigeninitiative der Beteiligten abhängig, ob sie ihr Projekt auf der Plattform präsentieren, und zum anderen sollte auch die Anzahl der Studiengänge, die hinter diesen Fächergruppen stehen, berücksichtigt werden.

Darüber hinaus werden für einzelne digitale Lehr-Lernformate oder -Ansätze Entwicklungslinien in und zwischen den Fächern untersucht. So ist der

\footnotetext{
${ }^{1}$ Digital Learning Map. https://www.e-teaching.org/community/digital-learning-map. Zugegriffen: 28.07.2020.

${ }^{2}$ Die hier dargestellten Fächergruppen folgen der auf der Projekthomepage dargestellten Clusterung und unterscheiden sich daher von der weiter unten gewählten Fächerklassifikation des Statistischen Bundesamtes.
} 
Gamification-Ansatz (Deterding et al. 2011) im Wesentlichen in den Bereichen IT und Informatik im Jahr 2008 entwickelt und erst im Jahr 2010 in weiteren Bereichen erprobt worden (Dicheva et al. 2015). Ferner konnte gezeigt werden, dass Blended-Learning-Ansätze einen Ursprung in der Germanistik haben (Hoyer und Mundt 2017) und später in anderen Linguistiken und in Psychologie und Soziologie Anwendung fanden (Kesselheim und Lindemann 2010). In den Rechtswissenschaften dominieren hingegen Wikis (Kaeding und Scholz 2012).

\section{$2 \quad$ Fragestellung}

Ziel dieses Artikels ist es, auf Basis einer Analyse von Publikationsdaten Entwicklungstrends in der Nutzung digitaler Formate, Methoden und technologischer Ansätze des Lehrens und Lernens an Hochschulen nachzuzeichnen und dabei insbesondere fachspezifische Unterschiede in den Blick zu nehmen. Wir vermuten, dass mit dem Ziel eines individualisierten und dem Lerngegenstand angemessenen Lernens verschiedene Fächer, entsprechend ihren „Fachkulturen“, verschiedene Zugänge zur Digitalisierung wählen und unterschiedliche digitale Formate und Technologien verwenden.

Mit unserem Beitrag bauen wir auf den oben beschriebenen Forschungsarbeiten auf und vergleichen Fachkulturen in Bezug auf ihren Umgang mit den Möglichkeiten der Digitalisierung. Dabei wählen wir einen bibliometrischen Zugang und gehen davon aus, dass mit diesem Zugang gefundene Unterschiede zwischen den Fächern im wissenschaftlichen Diskurs zumindest annäherungsweise auch die Unterschiede in der tatsächlichen Nutzung digitaler Lehr-Lernmethoden, -formate, -technologien sowie -tools abbilden.

Konkret sollen folgende Forschungsfragen untersucht werden:

1. Welche digitalen Lehr-Lernformate und -Methoden oder spezifischen Technologien werden im wissenschaftlichen Diskurs aufgegriffen und wie verbreitet sind diese jeweils?

2. Inwiefern unterscheiden sich Fächer in Bezug auf die primär im Fokus dieses Diskurses stehenden Lehr-Lernformate, -methoden und technologischen Ansätze (zum Beispiel virtuelle Labore, kollaborative Lernformate, adaptive Lernmodelle)? 


\section{$3 \quad$ Methode}

\subsection{Methodische Zugänge bei der Analyse fachspezifischer Besonderheiten}

Mit der hier zugrunde gelegten Methodik soll analysiert werden, in welchen Fächern und mit welchen Schwerpunkten Forschungsergebnisse zu Fragen des digitalen Lehrens und Lernens veröffentlicht wurden und welche inhaltlichen Trends erkennbar sind. Dies kann als Ausgangspunkt dienen, um weitere Untersuchungen, beispielsweise im Hinblick auf bestimmte Fächer oder Fachdisziplinen oder den Einsatz bestimmter digitaler Formate oder Lehr-Lernformen, gezielt zu untersuchen. Der vorliegende Abschnitt stellt die methodische Vorgehensweise einer solchen Analyse von Publikationsdaten vor.

\subsection{Datenquelle}

Die empirische Basis bilden Publikationsdaten aus der Literaturdatenbank Scopus. Scopus bietet bibliografische Angaben zu rund 200.000 Monografien, Sammelbänden, Forschungspapers, Patenten und Konferenzbeiträgen. Darüber hinaus sind über die Datenbank mehr als 75 Mio. Veröffentlichungen aus fast 25.000 Quellen auffindbar. $\mathrm{Zu}$ diesen Quellen zählen vor allem im Peer-Review-Verfahren veröffentlichte Fachzeitschriften. Die Auswahl dieser Datenquelle wurde vor allem deshalb vorgenommen, weil Scopus neben der großen Vielzahl an Dokumentenarten und der Abbildung einer großen Fächeranzahl auch sehr gute Analysemöglichkeiten bietet.

\subsection{Auswahl der Dokumente}

In Abb. 1 ist das methodische Vorgehen bei der Auswahl der in die Analyse einbezogenen Beiträge veranschaulicht: In einem ersten Schritt wurden verschiedene Suchbegriffe so miteinander verknüpft, dass möglichst alle Beiträge herausgefiltert wurden, die sich in Titeln, Abstracts oder Keywords mit dem Thema digitale 


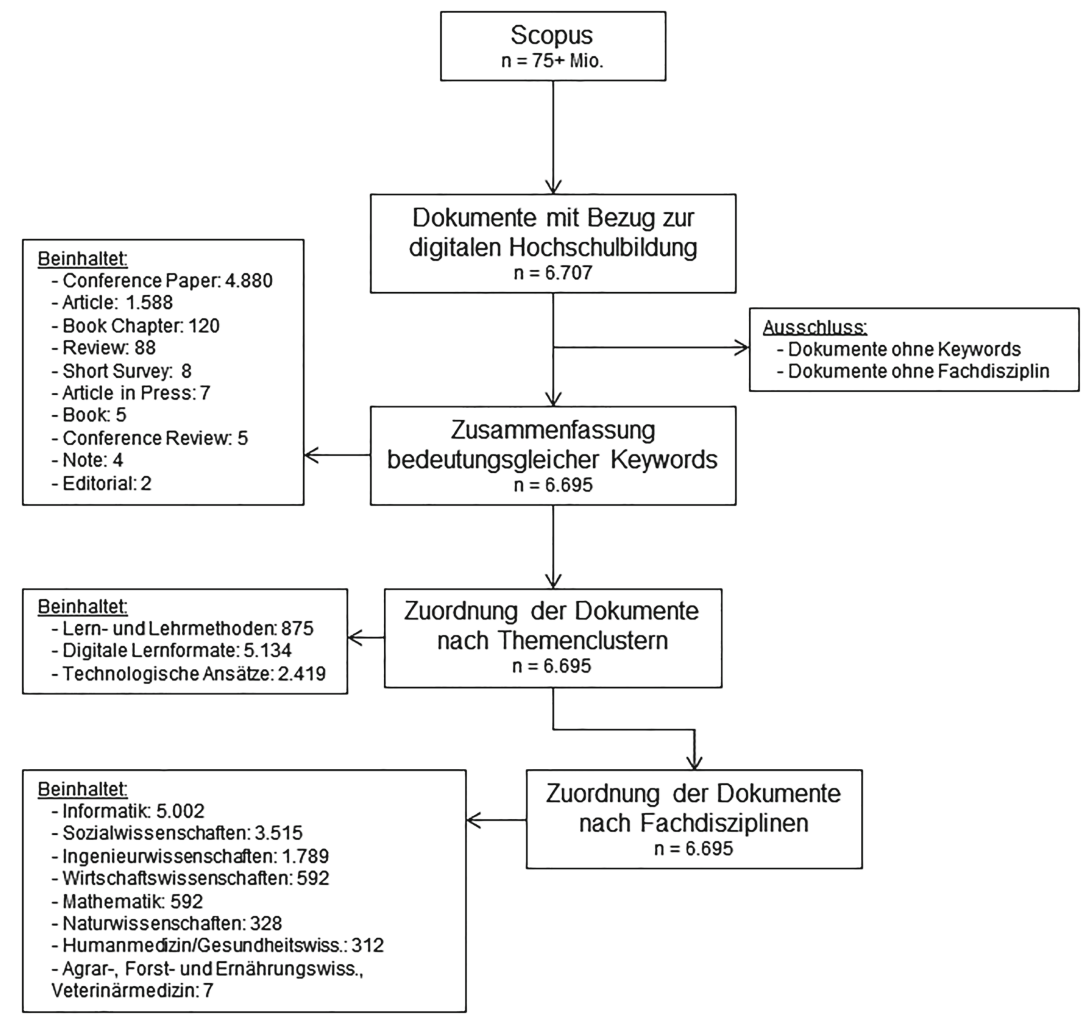

Abb. 1 Methodische Vorgehensweise. (Eigene Darstellung). In der Abbildung wurden die (englischen) Begrifflichkeiten aus Scopus übernommen, um eine eindeutige Reproduzierbarkeit der Ergebnisse sicherzustellen

Hochschulbildung befassen. ${ }^{3}$ Da unser Beitrag primär das Ziel verfolgt, den wissenschaftlichen Diskurs zum Thema in den einzelnen Fächern abzubilden, haben

${ }^{3}$ Der Export der Beiträge mit Bezug zur Hochschuldidaktik erfolgte am 12.3.2020, die Analysen wurden im Zeitraum März bis Juli 2020 durchgeführt. Ausgewählt wurden alle Publikationen, die in jedem Fall eines der drei Keywords higher education, tertiary education oder university education sowie ein Keyword enthalten, das digitale Bildung (zum Beispiel educational technology) oder eine didaktische Methode (zum Beispiel real-world learning) in Kombination mit ,digital“ oder „online“ beschreibt. Der genaue Suchstring kann von den Autor*innen zur Verfügung gestellt werden. 
sich die Autor*innen bewusst dafür entschieden, keine weitere Einschränkung bei der Auswahl der Dokumentenarten vorzunehmen. Eine reine Konzentration beispielsweise auf Beiträge mit Peer Review, wie sie häufig vorgenommen wird, wenn es darum geht, Effektstärken zu quantifizieren, hätte zum einen die Anzahl der Dokumente zu stark reduziert und zum anderen den wissenschaftlichen Diskurs nicht in jedem Fach adäquat abgebildet. So ist $\mathrm{zu}$ vermuten, dass für viele Fächer - insbesondere kleinere Fächer oder Fächer, deren primärer Forschungsgegenstand weder Digitalisierung noch Didaktik betrifft - nur eine sehr geringe Fallzahl von hochschuldidaktischen Publikationen mit Peer Review vorliegt. Wenn in diesem Fächern zu den genannten Themen publiziert wird, dann dürfte dies eher niedrigschwellig, also beispielsweise in Buch- oder Konferenzbeiträgen, passieren. Die weitere Analyse nach Lehr-Lernmethoden, -formaten sowie -technologien erfolgte nach den für die einzelnen Publikationen vergebenen Index Keywords.

\subsection{Bedeutung von Keywords}

Keywords (Schlagworte) sind ein etabliertes Forschungsobjekt: Mit ihrer Hilfe lassen sich wissenschaftliche Netzwerke darstellen und sie bilden zuverlässig Forschungsinhalte und -traditionen ab (zum Beispiel im Bereich Technik: Lee und Jang 2017; Philosophie: Barik und Jena 2014). ${ }^{4}$ Für die weitere Analyse wurden zunächst bedeutungsgleiche Keywords zusammengefasst (zum Beispiel MOOC, Massive Open Online Course und MOOCs). Aus der Analyse ausgeschlossen wurden ferner jene Ergebnisse, die keinem Fach und/oder keinem Keyword zugeordnet werden konnten. Aus diesen Arbeitsschritten ergab sich eine Analysebasis von 6695 Dokumenten und 17.065 unterschiedlichen Keywords (siehe Abb. 1).

\footnotetext{
${ }^{4}$ An dieser Stelle sei darauf hingewiesen, dass eine Analyse anhand von Keywords in der Wissenschaft durchaus kritisch gesehen wird, da ihre Auswahl im Publikationsprozess ein Teilschritt ist, der häufig nicht Teil der inhaltlichen Debatte ist (Rodrigues 2013). Seit 2020 fordert die American Psychological Association (2020) eine Strukturierung und Vereinheitlichung bei der Keywordvergabe in wissenschaftlichen Publikationen und unterstützt damit auch die Relevanz von Keywords. Für unseren Beitrag wäre eine eingeschränkte Sorgfalt bei der Auswahl vor allem dann ein Problem, wenn es Grund zu der Annahme gäbe, dass es in dieser Hinsicht systematische Unterschiede zwischen den Fächern gibt. Hierfür haben wir jedoch bislang keine Anhaltspunkte gefunden.
} 
Tab. 1 Zusammenfassung von Keywordgruppen zu Clustern. (Eigene Darstellung)

\begin{tabular}{l|l|l}
\hline Lern- und Lehrmethoden & Digitale Lernformate & Technologische Ansätze \\
\hline Experiential learning & Computer aided instruction & Virtual learning environment \\
\hline Game-based learning & Virtual learning & E-learning systems \\
\hline Problem-based learning & Online teaching & Learning technology \\
\hline Instructional designs & E-learning & Open educational resources \\
\hline E-portfolios & Online education & Moodle \\
\hline Active learning & Technology enhanced learning & Augmented reality \\
\hline Gamification & Mobile learning & Artificial intelligence \\
\hline Problem solving & MOOC & Multimedia systems \\
\hline Flipped classroom & Online learning & Virtual learning environments \\
\hline Collaborative learning & Blended learning & Learning systems \\
\hline Personnel training & Education computing & \\
\hline & &
\end{tabular}

\subsection{Analysemethode}

In einem ersten Analyseschritt wurden alle verwendeten Keywords den drei Kategorien Lern- und Lehrmethoden, digitale Lernformate und technologische Ansätze zugeordnet. Jedes Dokument wurde im Anschluss entweder einem oder mehreren Kategorien zugeordnet, da jedem Dokument mehrere Keywords entsprechen. Die Zuordnung der Keywords zu den drei Oberkategorien ist in Tab. 1 dargestellt.

\subsection{Differenzierung nach Fächergruppen}

Um die Unterscheidung in den Fächern abzubilden, wurden die Subject Areas aus Scopus (Scopus Fachgruppen) in Anlehnung an die Einteilung des Statistischen Bundesamtes (2018) in Fächergruppen überführt. Wie Tab. 2 zeigt, wurden die 27 Subject Areas in sieben Fächergruppen zusammengeführt: Sozialwissenschaften, Naturwissenschaften, Humanmedizin/Gesundheitswissenschaften, Mathematik, Ingenieurwissenschaften, Wirtschaftswissenschaften, Geisteswissenschaften. Die Subject Area „Multidisciplinary“ wurde keiner der Gruppen zugeordnet und wird in den Auswertungen nach Gruppen nicht beachtet.

Unter den analysierten Publikationen aus den Fächern Sozialwissenschaften und Informatik ist der Anteil interdisziplinärer, das heißt mehr als einer Subject Area zugeordneter Publikationen groß. Für die Sozialwissenschaften wurden mehr 
Tab. 2 Zuordnung von Subject Areas zu Fächergruppen. (Eigene Darstellung)

\begin{tabular}{l|l}
\hline Fächergruppen & Subject Areas \\
\hline Ingenieurwissenschaften & $\begin{array}{l}\text { Chemical Engineering, Energy, } \\
\text { Materials Science, Engineering, Computer Science }\end{array}$ \\
\hline Sozialwissenschaften & Psychology, Social Sciences \\
\hline Wirtschaftswissenschaften & $\begin{array}{l}\text { Economics, Econometrics and Finance, Business, } \\
\text { Management and Accounting, Decision Science }\end{array}$ \\
\hline Mathematik & Mathematics \\
\hline Naturwissenschaften & $\begin{array}{l}\text { Immunology and Microbiology, Neuroscience, Chemistry, } \\
\text { Pharmacology, Toxicology and Pharmaceutics, } \\
\text { Biochemistry, Genetics and Molecular Biology, Agricultural } \\
\text { and Biological Sciences, Earth and Planetary Sciences, } \\
\text { Physics and Astronomy, Environmental Science }\end{array}$ \\
\hline $\begin{array}{l}\text { Humanmedizin/ } \\
\text { Gesundheitswissenschaften }\end{array}$ & Dentistry, Health Professions, Nursing, Medicine \\
\hline Geisteswissenschaften & Arts and Humanities \\
\hline
\end{tabular}

als $90 \%$ der Publikationen in interdisziplinären Settings und für die Informatik wurden drei Viertel interdisziplinär veröffentlicht (siehe Tab. 3). Diese Verteilung wurde so erwartet, weil Sozialwissenschaften und Informatik im Themenschwerpunkt der Methode gleichzeitig Freiheiten in der Adressierung der Inhaltsbereiche bieten. Bei einer Fokussierung auf Publikationen, die ausschließlich in einer Fachdisziplin publiziert wurden, würde somit ein Großteil der Veröffentlichungen vernachlässigt werden.

Tab. 3 Verteilung der Publikationen nach der Anzahl der beteiligten Disziplinen. (Eigene Darstellung)

\begin{tabular}{l|l|l|l|l}
\hline & \multicolumn{3}{l|}{ Sozialwissenschaften } & \multicolumn{2}{l}{ Informatik } \\
\hline Nicht interdisziplinär & 254 & $7,2 \%$ & 1262 & $25,2 \%$ \\
\hline $\begin{array}{l}\text { Interdisziplinär (2 } \\
\text { Subject Areas) }\end{array}$ & 2439 & $69,4 \%$ & 2886 & $57,7 \%$ \\
\hline $\begin{array}{l}\text { Interdisziplinär (3 } \\
\text { Subject Areas) }\end{array}$ & 778 & $22,1 \%$ & 775 & $15,5 \%$ \\
\hline $\begin{array}{l}\text { Interdisziplinär (4 } \\
\text { Subject Areas) }\end{array}$ & 38 & $1,1 \%$ & 73 & $1,5 \%$ \\
\hline $\begin{array}{l}\text { Interdisziplinär (5 } \\
\text { Subject Areas) }\end{array}$ & 6 & $0,2 \%$ & 6 & $0,1 \%$ \\
\hline
\end{tabular}




\subsection{Exploratives Untersuchungsdesign}

Aufgrund der explorativen Anlage der Studie werden im Folgenden ausschließlich deskriptive Statistiken berücksichtigt. Dies bietet die Möglichkeit, Unterschiede in den Fächerkulturen zu beschreiben, auch wenn sie vergleichsweise klein sind. Eine Beschränkung der Daten auf Fächer mit einer Mindestfallzahl wurde diskutiert und verworfen, da diese Fächer in diesem Fall nicht hätten berücksichtigt werden können. Die Autor*innen sind sich der Einschränkungen dieses Vorgehens bewusst: Statistische Signifikanztests können für die Studien nicht sinnvoll angewendet werden, die Analyse soll vielmehr ein erster Schritt sein, um Hypothesen $\mathrm{zu}$ generieren und weitere Forschungsarbeiten zu stimulieren.

\section{$4 \quad$ Ergebnisse}

Im Folgenden werden die Ergebnisse der Literaturanalyse dargestellt. Dabei gehen wir zunächst auf die generelle Verbreitung und Entwicklung technologischer Ansätze, digitaler Lehr-Lernformate und -Methoden ein (Abschn. 4.1), bevor in Abschn. 4.2 eine Differenzierung nach Fächern vorgestellt wird.

\subsection{Verbreitung und Entwicklung technologischer Ansätze, digitaler Lehr-Lernformate und -methoden}

Bei der Analyse der Beiträge zur digitalen Hochschulbildung wurde die oben vorgestellte Kategorisierung in technologische Ansätze, digitale Lernformate und Lern- und Lehrmethoden beibehalten. Dementsprechend folgt auch die Darstellung der Entwicklungslinien dieser Aufteilung.

\subsubsection{Technologische Ansätze}

Eine Analyse der Häufigkeiten unterschiedlicher technologischer Ansätze zeigt zunächst, dass sich ein Großteil der Beiträge vor allem zwei Ansätzen widmet (siehe Abb. 2): Von den insgesamt 2419 Beiträgen behandelt mehr als die Hälfte $(1338,55 \%)$ learning systems und ein Drittel $(799,33 \%)$ behandelt virtual learning environments. Betrachtet man die Zahl der Dokumente, die sich mit technologischen Ansätzen beschäftigen, im Zeitverlauf (siehe Abb. 3), so zeigt sich: Erste Nennungen treten 1995 auf; ein deutlicher Anstieg findet aber erst ab dem Jahr 2005 statt, was vermutlich die neuen Möglichkeiten des Web 2.0 ab 2003 widerspiegelt. Zwischen 2005 und 2019 hat sich die Zahl der Dokumente mit 


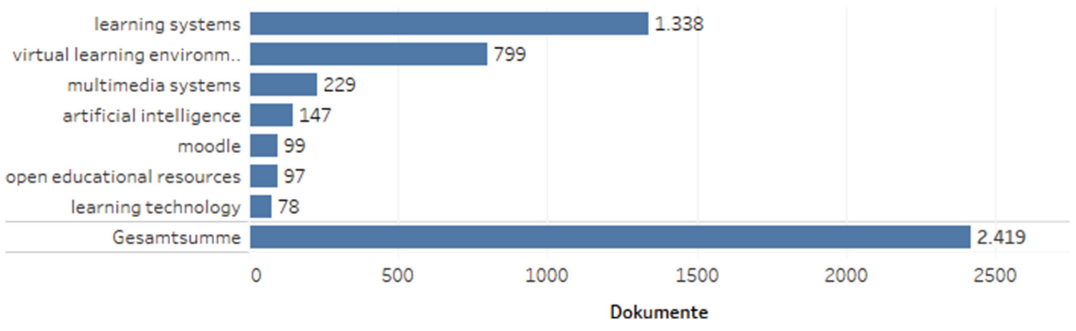

Abb.2 Technologische Ansätze, Dokumente mit entsprechenden Keywords nach Häufigkeit. (Eigene Darstellung)

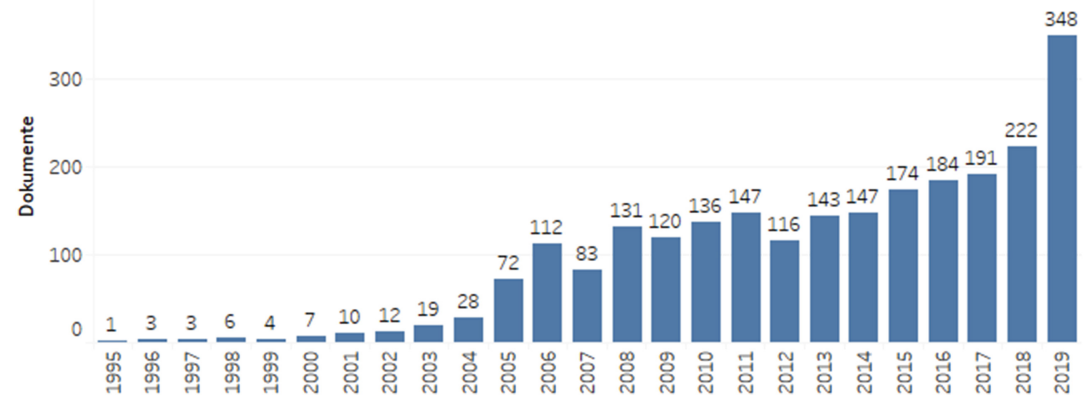

Abb.3 Technologische Ansätze, Entwicklung im Zeitverlauf. (Eigene Darstellung)

entsprechenden Keywords deutlich erhöht. Der Anstieg seit 2005 kann in erster Linie durch das gehäufte Auftreten der beiden häufigsten Keywords learning systems und virtual learning environments seit dem Jahr 2005 erklärt werden. Andere Keywords setzen interessanterweise erst später ein: Artifical intelligence etwa hält als Thema erst ab dem Jahr 2013 Einzug in den wissenschaftlichen Diskurs. Zuvor sind nur vereinzelte Nennungen dieses Begriffs zu verzeichnen.

\subsubsection{Digitale Lernformate}

Bei den digitalen Lernformaten dominiert der Begriff e-learning den wissenschaftlichen Diskurs (4220 von 5134 Dokumenten; also 82 \%). Dahinter folgen mit großem Abstand computer aided instruction (1154 Dokumente, $22 \%$ ) und education computing (960 Dokumente, $18 \%$; siehe Abb. 4). Das Keyword $e$ learning taucht zwar bereits 1987 das erste Mal in einem hochschuldidaktischen 


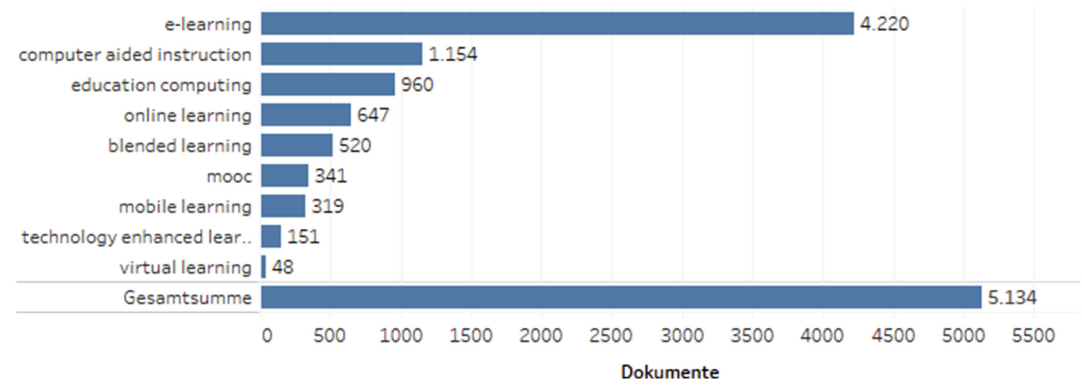

Abb. 4 Digitale Lernformate, Dokumente mit entsprechenden Keywords nach Häufigkeit. (Eigene Darstellung)

Beitrag auf, allerdings verbreitet sich der Begriff erst deutlich später. Während die Scopus-Analyse für 2004 nur 24 Beiträge mit diesem Bezug ausweist, hat sich die Zahl im Jahr 2005 mit 92 Dokumenten fast vervierfacht. Für das Jahr 2019 werden wiederum 531 Dokumente diesem Keyword zugeordnet. Für computer aided instruction lässt sich ein ähnlicher zeitlicher Verlauf aufzeigen. Der Begriff Massive Open Online Courses (MOOCs) findet erst im Jahr 2012 Einzug in den Diskurs und steigt danach stark an, was den Boom in der Entwicklung und Durchführung von MOOCs nach der Publikation eines Artikels in der New York Times (Pappano 2012) widerspiegelt, der zu einer enormen medialen Aufmerksamkeit führte. Seitdem hat sich der Begriff stark verbreitet und wird 2019 bereits in $6 \%$ (341) der Dokumente als Keyword genannt. Abḅ. 5 zeigt eine Übersicht über die Entwicklung der digitalen Lernformate.

\subsubsection{Lern- und Lehrmethoden}

Bei den digitalen Lern- und Lehrmethoden unterscheiden sich die Keywords nach Häufigkeiten weniger stark als bei den zuvor beschriebenen Ansätzen. Collaborative learning wird am häufigsten als Keyword vergeben: in 232 von 875 Dokumenten (27\%). Dahinter folgen problem based learning mit Nennung in 175 Dokumenten (20\%) und gamification mit 144 Dokumenten (16\%; siehe Abb. 6). Wie der Zeitverlauf in Abb. 7 zeigt, gibt es auch bei den Lern- und Lehrmethoden einen deutlichen Anstieg der Anzahl der Dokumente seit Mitte der 2000er-Jahre, was auch hier wieder mit den Möglichkeiten des aufkommenden Web 2.0 zusammenhängen dürfte. Interessant ist, dass Lern- und Lehrmethoden insgesamt in den Keywords erstmals ab 2001 auftreten (collaborative learning mit Nennungen in zwei Dokumenten) und damit deutlich später als technologische Ansätze (ab 


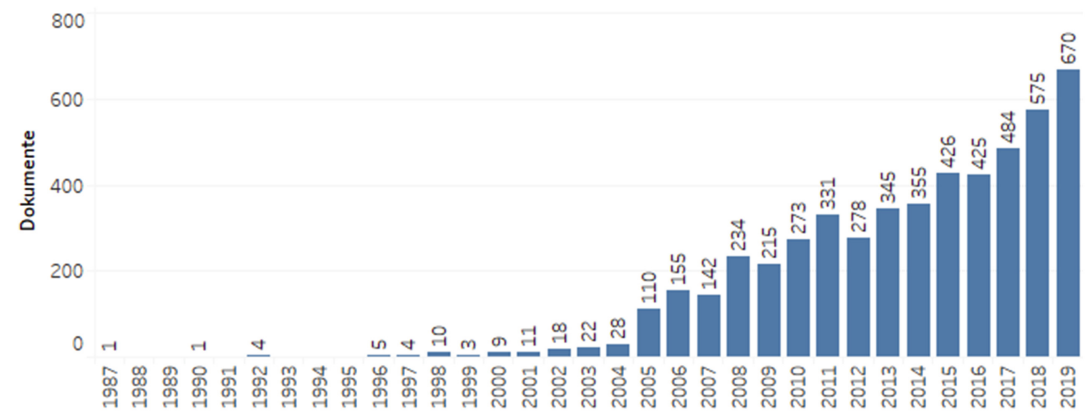

Abb. 5 Digitale Lernformate, Entwicklung im Zeitverlauf. (Eigene Darstellung)

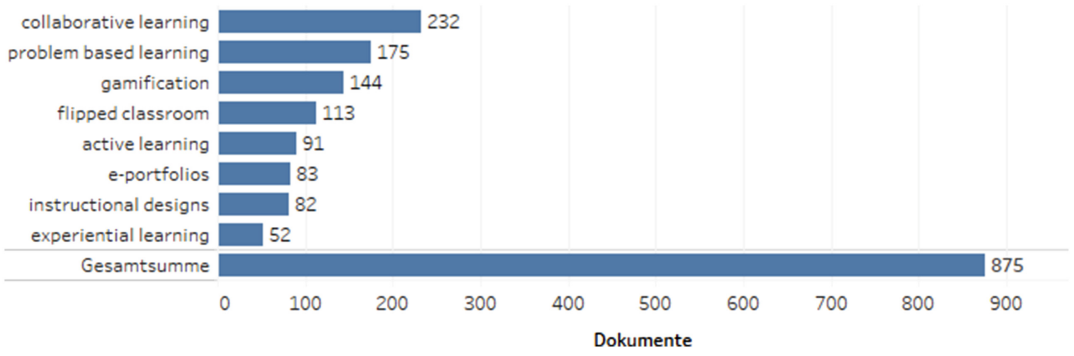

Abb. 6 Lern- und Lehrmethoden, Dokumente mit entsprechenden Keywords nach Häufigkeit. (Eigene Darstellung)

1995) und digitale Lernformate (ab 1987). Auffällig ist zudem, dass das Keyword flipped classroom erst 2012 auftritt und dann eine beachtliche Verbreitung erfahren hat, bis auf 113 Nennungen (kumuliert) im Jahr 2019. Analog zu dem Boom für die MOOCs findet sich auch für den flipped classroom ein ähnlicher Anstieg in den Publikationen aufgrund einer populären Veröffentlichung. 5

${ }^{5} 2012$ wurde „Flip your classroom“ (Bergmann und Sams 2012) veröffentlicht und hat mit ca. 5000 Zitationen (vgl. Google Scholar) eine beachtliche Reichweite erlangt. 


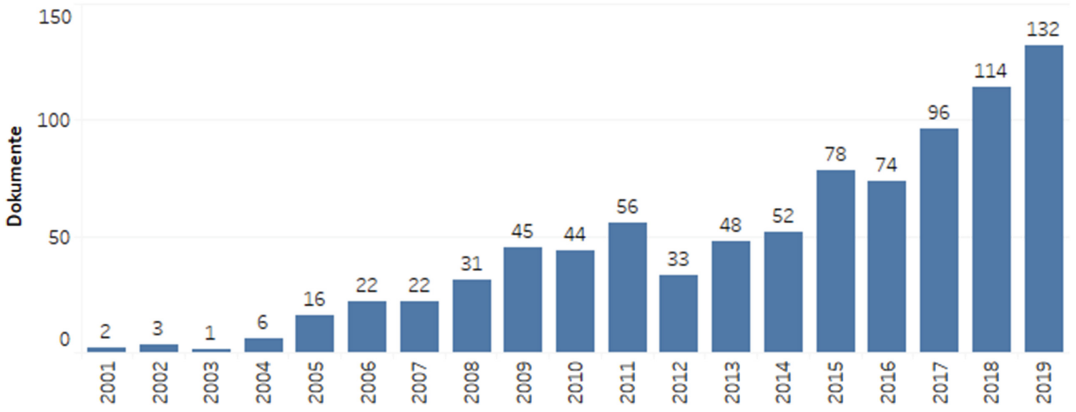

Abb. 7 Lern- und Lehrmethoden, Entwicklung im Zeitverlauf. (Eigene Darstellung)

\subsection{Digitale Lehr-Lernformate, -methoden und technologische Ansätze im Fächervergleich}

Nach der Auswertung der Publikationen im Hinblick auf Verbreitung und Entwicklung der Keywords wird nun eine fächervergleichende Perspektive eingenommen. Bei einer Gesamtbetrachtung der Ergebnisse zu Lehr-Lernmethoden, technologischen Ansätzen sowie Lernformaten zeigt sich durchgängig, dass die meisten Publikationen in den Sozialwissenschaften und der Informatik zu finden sind (siehe Abb. 8).

Informatik und sozialwissenschaftliche Fächer wie Pädagogik und Erziehungswissenschaften sind dabei die Fächer, die sich aus fachwissenschaftlicher Sicht mit Fragen des digitalen Lehrens und Lernens beschäftigen - damit muss aber keineswegs die Lehre im eigenen Fach im Fokus der Untersuchung stehen, genauso gut kann hier der Fokus fachübergreifend oder auf jedes beliebige Fach ausgerichtet sein. Die hohe Anzahl an Publikationen in diesem Bereich sollte also nicht so interpretiert werden, dass diese Fächer in höherem Maße digitale Lehre praktizieren. Vielmehr ist in ihrer Fachwissenschaft digitale Hochschulbildung ein 


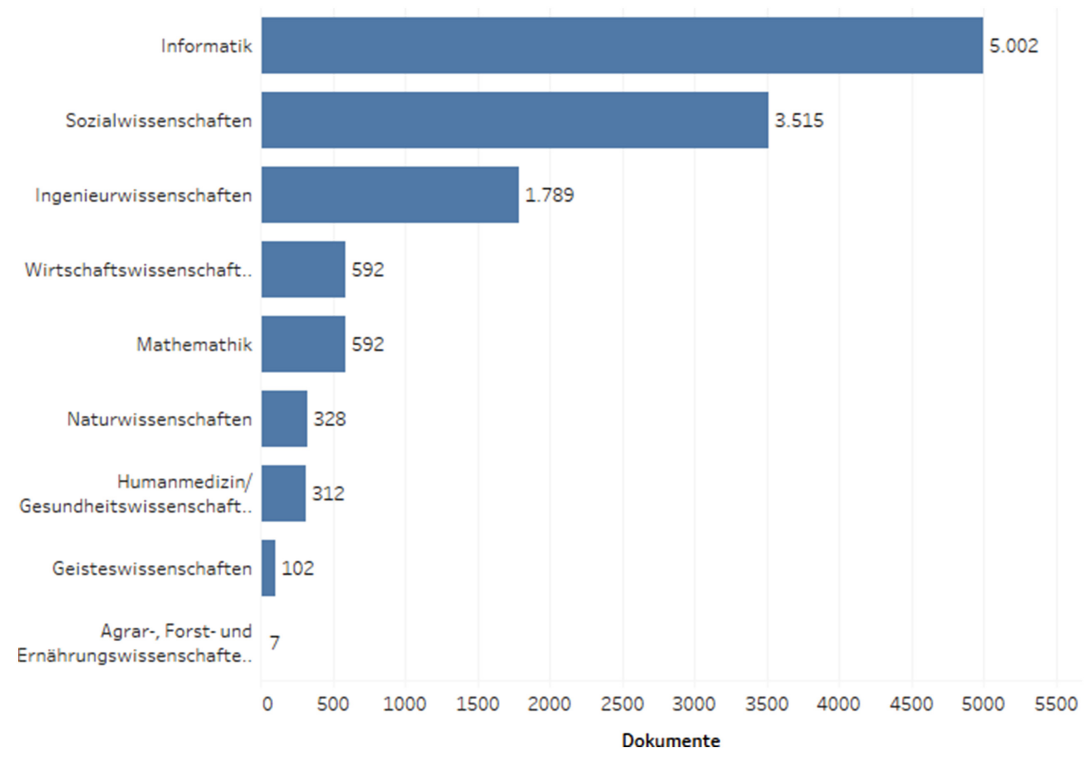

Abb. 8 Anzahl der Dokumente nach Fächergruppen. (Eigene Darstellung)

Untersuchungsgegenstand. ${ }^{6}$ Dennoch ist der Anteil an Publikationen in anderen Fachdisziplinen im Vergleich zu anderen Forschungsgegenständen relativ groß.

Wie in Tab. 4 dargestellt, zeigen sich im Fächervergleich bei den LehrLern-Methoden erstaunlich wenig Unterschiede, das heißt, die Anteile, die die einzelnen Keywords bzw. Lehr-Lern-Methoden jeweils innerhalb der Fächer beanspruchen, sind im Vergleich sehr ähnlich. Lediglich punktuell lassen sich Unterschiede aufzeigen: Kollaborative Ansätze werden mit circa $30 \%$ anteilig am stärksten in den Wirtschaftswissenschaften, der Informatik und den Sozialwissenschaften diskutiert. Eine deutliche Mehrheit (60\%) der lehrmethodischen

\footnotetext{
${ }^{6}$ Auch ein Blick auf laufende Forschungsprojekte zum Thema digitale Hochschulbildung - gut möglich am Beispiel der Projekte, die vom BMBF im Forschungsfeld Digitale Hochschulbildung innerhalb des Förderschwerpunkts ,Wissenschafts- und Hochschulforschung “ gefördert werden - bestätigt diese Perspektive: In vielen Projekten arbeiten Fachwissenschaftler*innen aus Informationstechnologie/Informatik und aus dem pädagogischen Bereich interdisziplinär zusammen (siehe Projektübersichten auf https://www.wihoforschung.de/de/erste-foerde rlinie-zur-digitalen-hochschulbildung-2378.php sowie die dort verlinkten Projekthomepages. Zugegriffen: 16.09.2020).
} 
Publikationen in den Geisteswissenschaften beschäftigt sich mit dem kollaborativen Lernen, allerdings ist der Anteil an Publikationen aus dieser Fächergruppe insgesamt relativ gering. In der Mathematik ist hingegen der hohe Anteil an Gamification-Ansätzen auffällig (über $30 \%$ ). Weiter zeichnet sich dieses Fach durch einen überdurchschnittlichen Anteil (circa $20 \%$ ) an Publikationen im Bereich des instructional design aus. In den Fächergruppen Naturwissenschaften (circa $40 \%$ ) und Humanmedizin/Gesundheitswissenschaften (circa $70 \%$ ) wird eher zu problembasierten Lernstrategien publiziert (wobei auch dieser Befund auf sehr kleinen Fallzahlen basiert). Die Konzepte flipped classroom und e-portfolios werden über alle Fächer hinweg in etwa zu gleichen Anteilen herangezogen. Kleinere Unterschiede zeigen sich auch bei Publikationen zu Formen des aktiven oder experimentellen Lernens, die tendenziell eher in den Ingenieur-, Wirtschafts- und Naturwissenschaften referiert werden.

Die Auswertung der Publikationen, die sich mit technologischen Ansätzen beschäftigen (siehe Tab. 5), zeigt, dass am häufigsten zu Keywords publiziert wird, die eher umfassend sind und die Lernumgebung als Ganzes begrifflich erfassen (learning systems, virtual learning environments und multimedia systems). Bei diesen drei Begriffen lassen sich keine bedeutsamen Unterschiede zwischen den Fächern ausmachen. Ein Schwerpunkt für das virtuelle Lernen scheint sich in den Naturwissenschaften abzuzeichnen, in denen jede zweite Publikation diesen Bereich adressiert. Danach folgt der Begriff artificial intelligence mit 148 Artikeln. Dieser Begriff wird in den Naturwissenschaften und der Mathematik häufiger verwendet als in der Informatik, den Sozial- und den Ingenieurwissenschaften.

Tab. 6 zeigt die Analyse der Lernformate im Fächervergleich. Hierbei ist für das Keyword computer aided instruction eine vergleichsweise häufige Verwendung (circa $55 \%$ ) in den Geisteswissenschaften auffällig. Hier wäre es interessant, in zukünftigen Forschungsprojekten die Entstehung und Verbreitung des Begriffs innerhalb der Geisteswissenschaften nachzuvollziehen (vgl. Kap. 2). Auch für das Keyword technology enhanced learning zeigt sich in den Geisteswissenschaften im Vergleich zu anderen Fächern ein etwas höheres Vorkommen.

Für die Fächergruppen Informatik, Sozial- und Ingenieurwissenschaften zeichnen sich im Bereich der Lernformate keine deutlichen Schwerpunkte ab; ein Drittel der Publikationen wird jeweils zu computer aided instruction sowie zu education computing verfasst. Der Begriff education computing wird in den Wirtschaftswissenschaften und den Geisteswissenschaften vergleichsweise häufig (mehr als ein Drittel der Publikationen) zitiert. Blended learning scheint als Lernformat eher etabliert zu sein und wird über alle Fächer hinweg in vergleichbaren Anteilen (circa $15 \%$ ) referiert. Auffällig ist zudem, dass Publikationen 
Fächerunterschiede in der digitalen Hochschulbildung ...

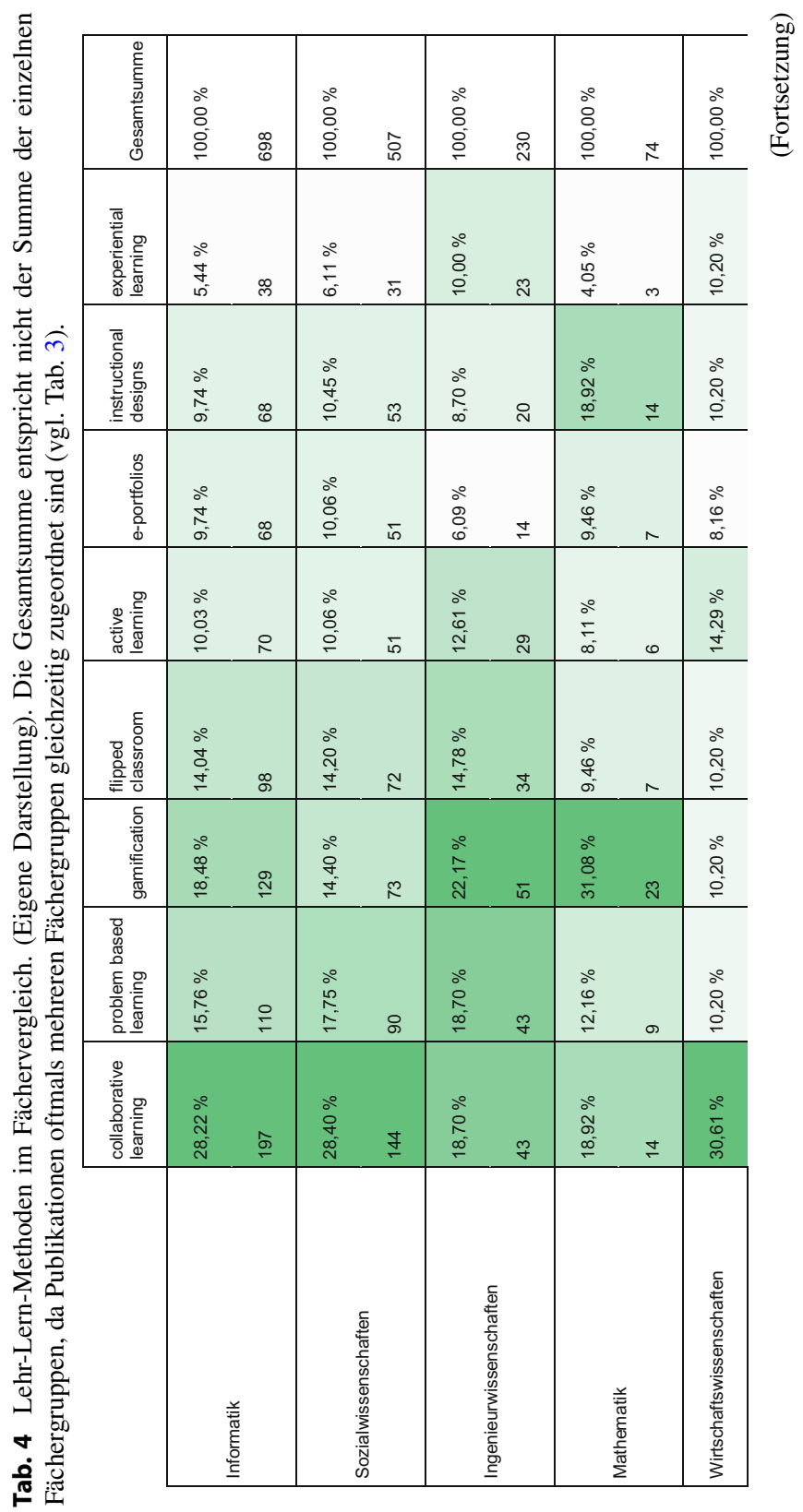




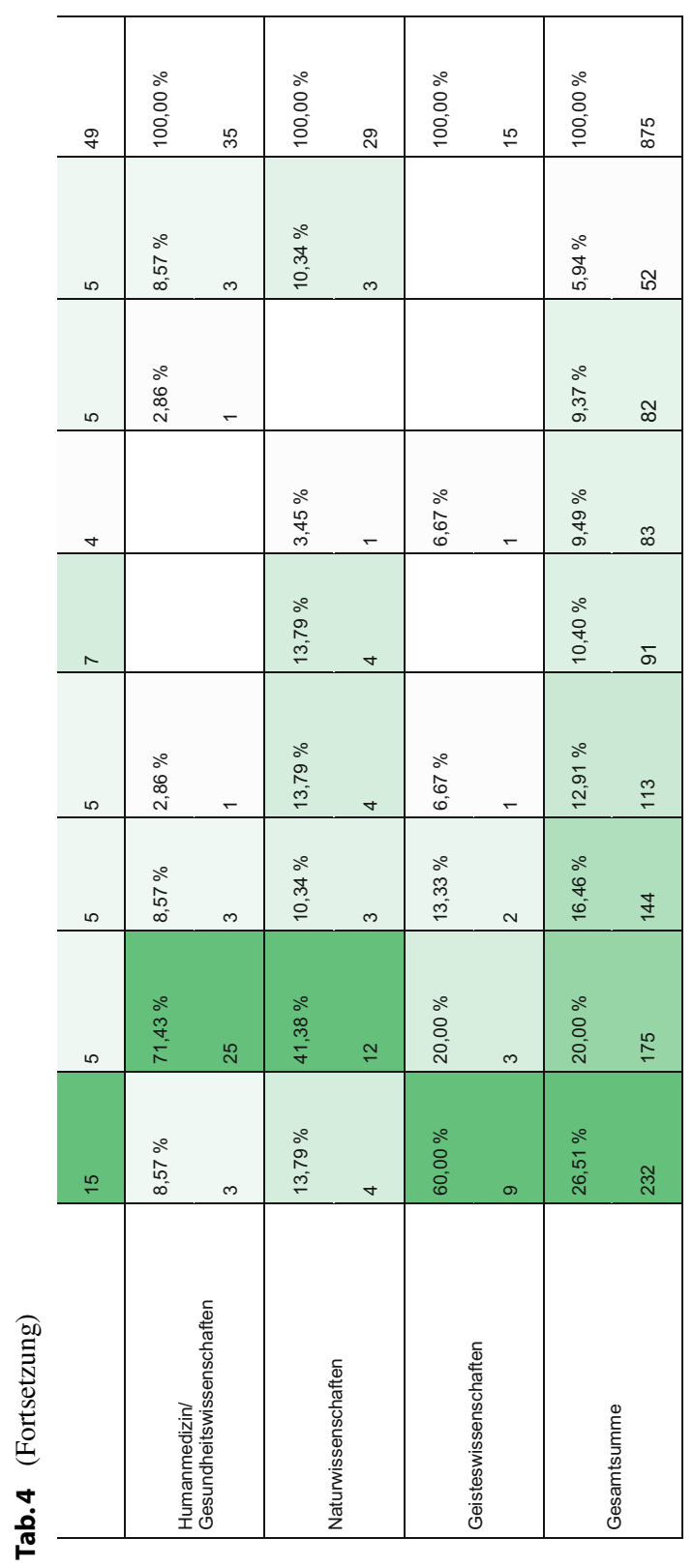




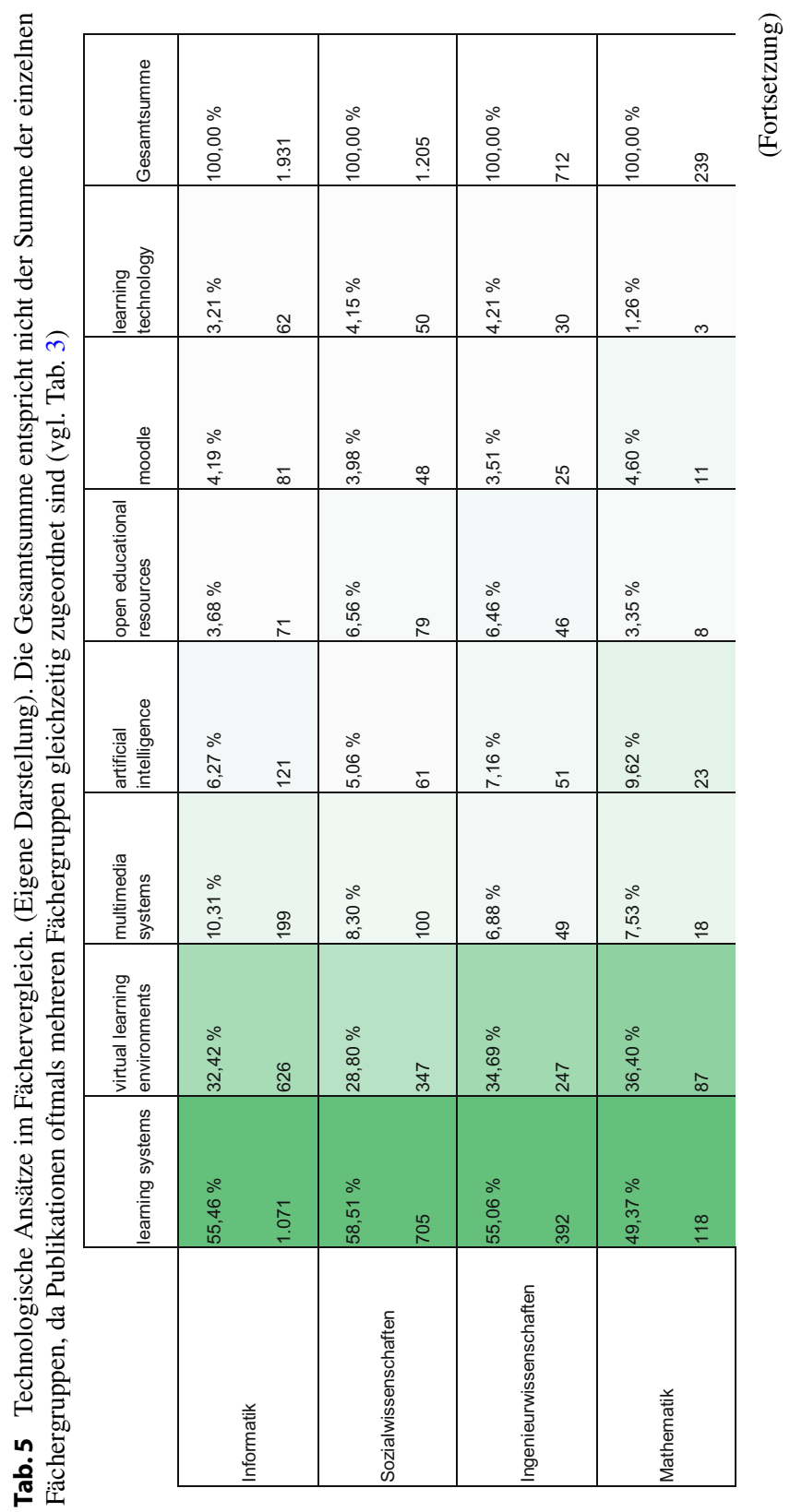




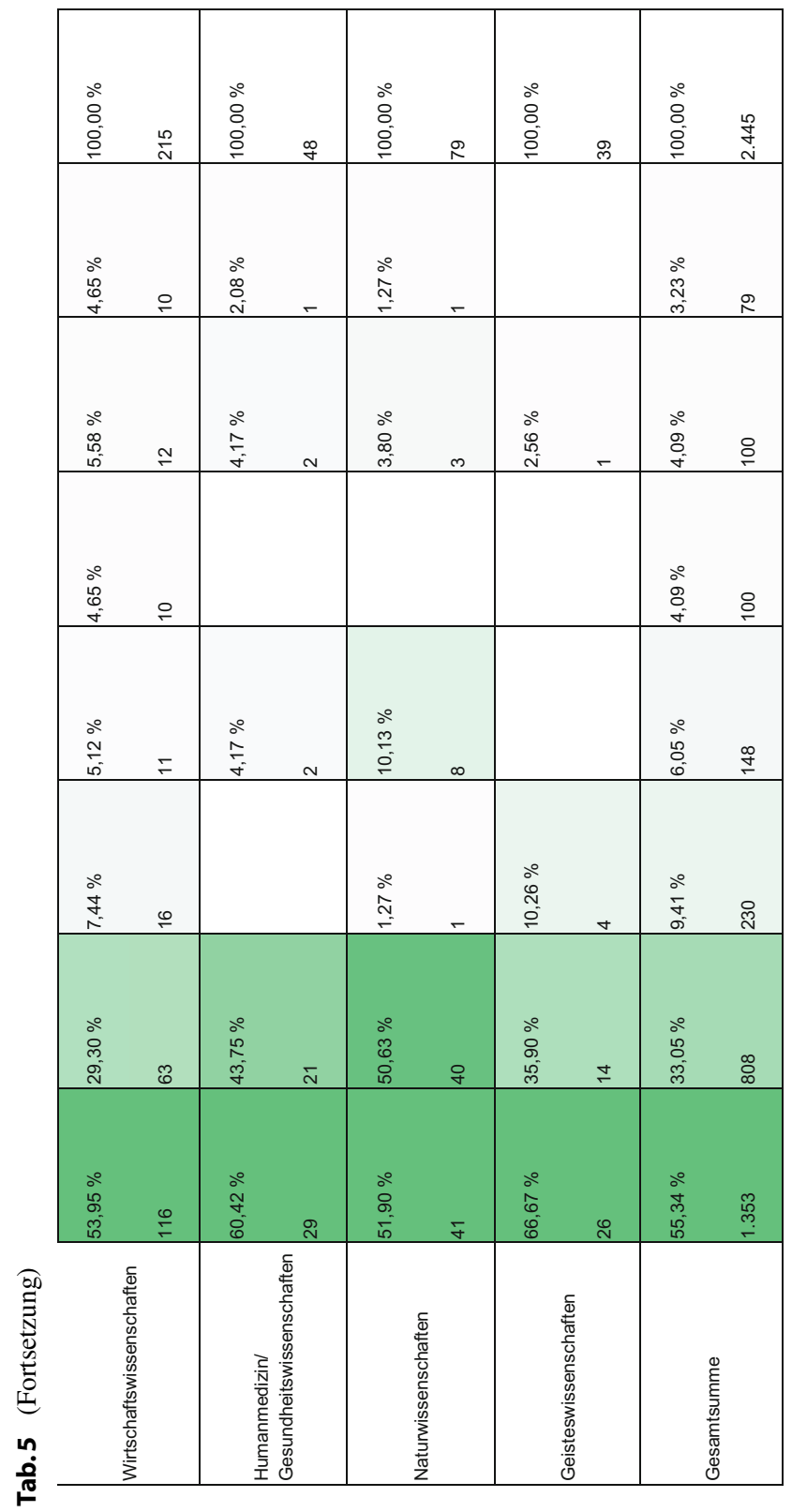




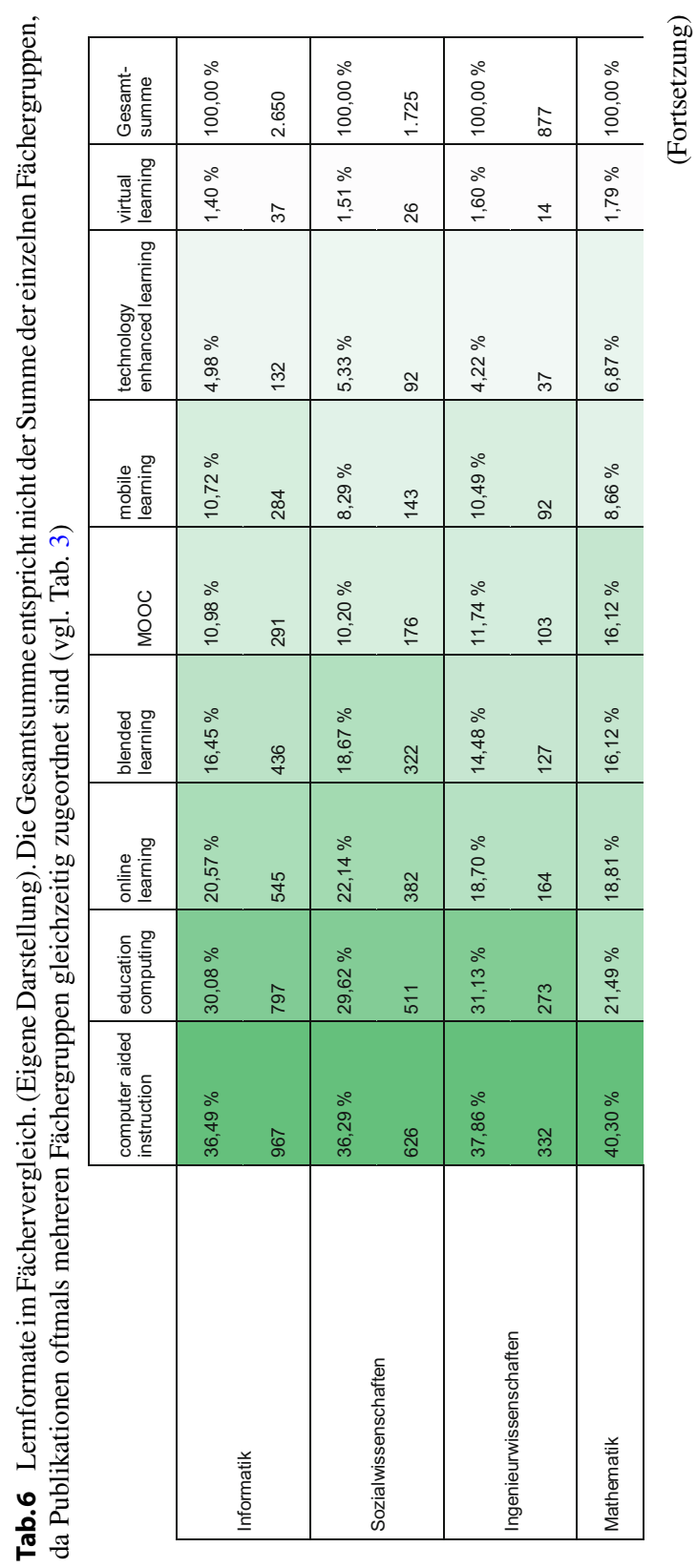




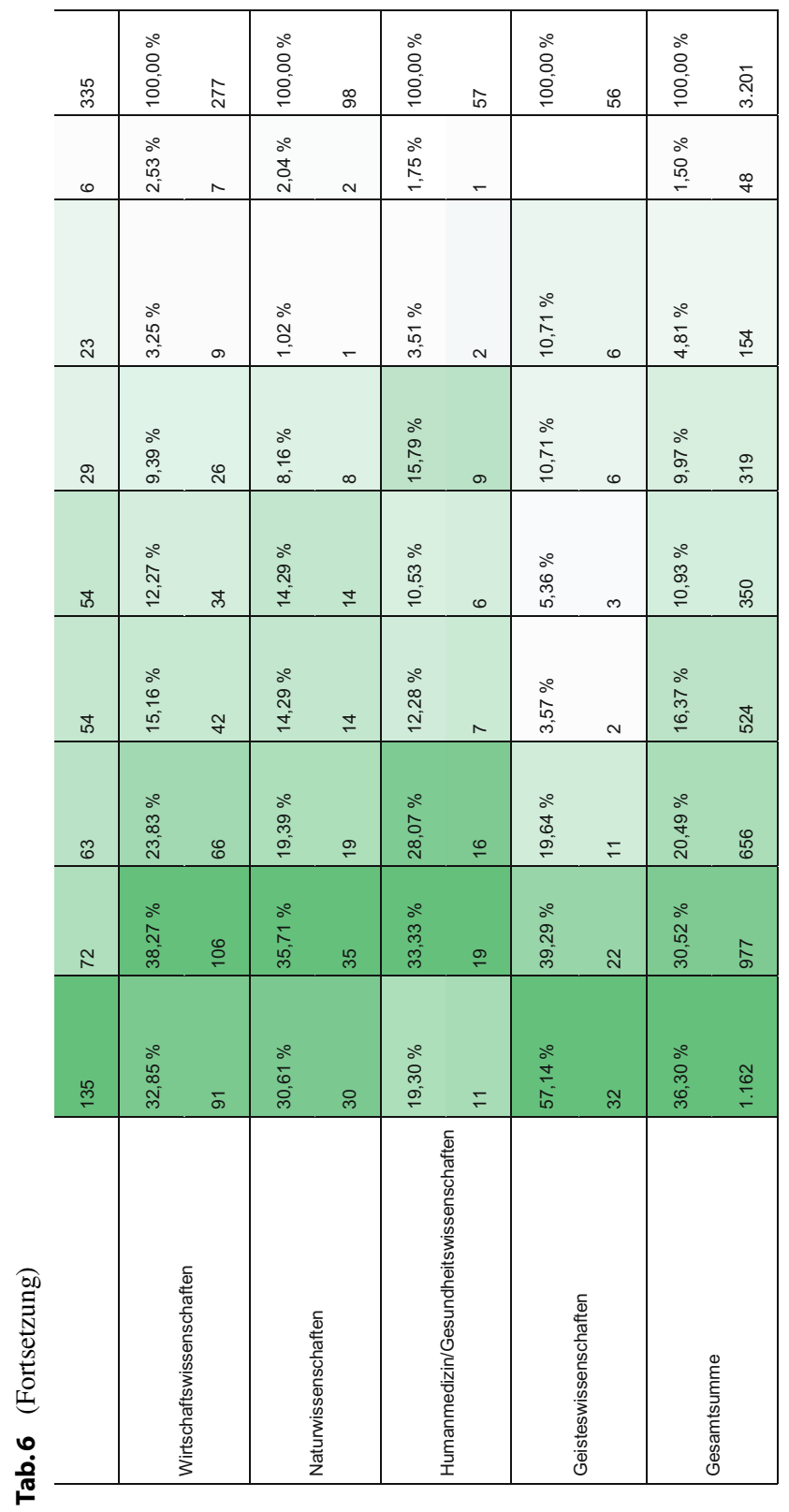


zu MOOCs in der Mathematik (circa $15 \%$ ) und den Naturwissenschaften (circa $13 \%$ ) häufiger vorkommen als in anderen Fachdisziplinen. Für das mobile learning zeigt sich ein Schwerpunkt in der Gesundheitswissenschaft mit etwa einem Sechstel der Publikationen.

\section{Diskussion}

Ziel dieses Beitrages war es, auf der Grundlage einer systematischen Literaturanalyse zu untersuchen, welche digitalen Lehr-Lernformate und -Methoden sowie spezifischen Technologien im wissenschaftlichen Diskurs aufgegriffen werden, wie verbreitet diese jeweils sind und inwiefern sich Fächer in Bezug auf die primär im Fokus stehenden Ansätze unterscheiden. Wie oben beschrieben, zeigt sich hier zunächst, dass in Publikationen, die sich mit technologischen Ansätzen beschäftigen, am häufigsten zu Keywords publiziert wird, die eher umfassend sind und die Lernumgebung als Ganzes begrifflich erfassen. Auch die Diskussion zu digitalen Lernformaten wird von den globalen Begriffen e-learning und computeraided instruction dominiert. Bei den digitalen Lehr-Lern-Methoden zeigt sich hingegen eine größere Vielfalt.

Unsere Analyse konnte punktuelle Unterschiede zwischen den Fächergruppen im wissenschaftlichen Diskurs zur digitalen Hochschulbildung aufzeigen. Beispielsweise sind Publikationen zu MOOCs besonders im Bereich der Mathematik und Naturwissenschaften zu finden. Gamification ist besonders in den Ingenieurwissenschaften ein Thema und problem based learning insbesondere im Bereich Humanmedizin/Gesundheitswissenschaften. Diese Unterschiede erscheinen durchaus plausibel. So ist beispielsweise anzunehmen, dass die klassischen Lehr-Lern-Methoden in den Geisteswissenschaften durchaus kooperativer sind als in den Naturwissenschaften, weshalb Letztere weniger Interesse an Konzepten des collaborative learnings haben. In weiteren Forschungsarbeiten wäre es interessant, genauer auszuloten, inwieweit sich im Einsatz digitaler Lehr-Lern-Methoden die didaktischen Traditionen der einzelnen Fächer widerspiegeln.

Insgesamt zeigen sich zwischen den Fächern jedoch deutlich weniger Unterschiede als erwartet.

Die Ergebnisse lassen sich zunächst so interpretieren, dass eine auf die ,Inhalte der einzelnen Fachdisziplinen bezogene, dem jeweiligen Thema angemessene Nutzung digitaler Medien“ (KMK 2019, S. 11) bislang noch relativ wenig stattfindet und die Chancen der Digitalisierung, die sich insbesondere in der Verknüpfung mit den Besonderheiten der jeweiligen Fachkultur ergeben, noch nicht genügend 
ergriffen werden. In weiteren Forschungsarbeiten gilt es nun, diese besonderen Erfordernisse zu erfassen, mit den existierenden Ansätzen in den einzelnen Fächern abzugleichen, um schließlich die von der KMK geforderten fachbezogenen Lehrkonzepte für Lehrende zur Verfügung stellen zu können. Interessant ist zudem die Frage, wie der von der KMK ebenfalls geforderte Austausch innerhalb der Fächer funktioniert. Dabei sind wissenschaftliche Publikationen nur ein Weg, um Forschungsergebnisse zu teilen. Möglicherweise sind für diejenigen Akteur*innen, die in der Praxis der Hochschullehre tätig sind, andere Medien und Publikationsorgane eine wichtigere Informationsquelle. ${ }^{7}$ Birkenkrahe et al. (2019) gehen davon aus, dass Unterschiede zwischen Lehrenden im Umgang mit digitalen Technologen kleiner werden, wenn Informationen über digitale Ansätze im Fachbereich stark verbreitet sind. Inwiefern Lehrende digitale Lehre einsetzen, hängt also auch von der Verbreitung digitaler Ansätze in dem jeweiligen Fach ab. ${ }^{8}$

Abschließend sei jedoch noch darauf hingewiesen, dass der in diesem Beitrag gewählte methodische Zugang mit einigen methodischen Einschränkungen einhergeht. Zunächst ist die Anzahl der gefundenen Publikationen auf der Ebene der Fächer vergleichsweise gering, sodass wir die gefundenen Unterschiede nicht inferenzstatistisch absichern können. Hier erscheint es sinnvoll, in weiteren Forschungsarbeiten auszuloten, inwieweit andere oder auch die Kombination mehrerer Datenquellen höhere Fallzahlen liefern können. Es ist durchaus plausibel, dass Akteur*innen, die die digitale Lehre forschend und entwickelnd vorantreiben, in großen Teilen eher einen anwendungsbezogenen und praxisorientierten Ansatz nutzen, bei dem die Veröffentlichung in wissenschaftlichen Journalen weniger im Fokus steht. Es ist anzunehmen, dass etwaige Publikationen zudem eher wenig in den Fachzeitschriften erscheinen, die in Scopus abgebildet sind. Mit diesen möglichen Verzerrungen ließe sich im Übrigen auch begründen, warum die meisten Publikationen aus den Sozialwissenschaften und der Informatik kommen: Beide Disziplinen beschäftigen sich aus fachwissenschaftlicher Sicht mit Fragen des digitalen Lehrens und Lernens und hochschuldidaktische Themen werden nicht zusätzlich zum eigentlichen Forschungsgebiet behandelt, sondern stellen den eigentlichen Forschungsgegenstand dar. In diesem Kontext könnte

\footnotetext{
${ }^{7}$ Ein Beispiel für ein solches Austauschorgan könnte die Gesellschaft für Medien in der Wissenschaft (GMW) werden, die als Fachgesellschaft für Fragen des Medieneinsatzes an Hochschulen wesentlichen Einfluss nehmen kann.

${ }^{8}$ Ein Faktor, der aktuell die Adaption digitaler Lehre beeinflusst, ist natürlich die durch das Coronavirus bedingte Situation, die Lehrende zum Einsatz digitaler Technologie zwingt. Vermutlich sind die dadurch angestoßenen Entwicklungen aber weniger fächerspezifisch, sondern hochschulübergreifend ausgerichtet.
} 
eine weiterführende vergleichende Analyse verschiedener Datenbanken ebensolche Unterschiede in den Publikationsstrategien aufzeigen und auch Verzerrungen entgegenwirken, die mit einer kommerziellen und auf bestimmte Zeitschriften konzentrierten Datenbank wie Scopus einhergehen.

Unsere Analysen stellen also nur einen ersten Schritt dar, um den Status quo der digitalen Hochschullehre in den einzelnen Fächern abzubilden. Dabei spricht jedoch die Tatsache, dass sich sowohl bei den MOOCs als auch beim flipped classroom die jeweiligen bekannten zeitlichen Hochphasen infolge populärer Veröffentlichungen in unseren Daten abbilden, für deren Validität.

\section{Literatur}

American Psychological Association. (2020). Publication manual of the American psychological association, (7th ed.). 10.10370000165.000.

Barik, N., \& Jena, P. (2014). Growth of LIS research articles in India seen through scopus: A bibliometric analysis. Library Philosophy and Practice (e-journal), 1133.

Bergmann, J., \& Sams, A. (2012). Flip your classroom: Reach every student in every class every day. Eugene: International Society for Technology in Education, ASCD.

Birkenkrahe, M., Hingst, A., \& Mey, S. (2019). „Ja, ich will.“Wie können Lehrende für die digitale Transformation begeistert werden? In S. Robra-Bissantz, O. J. Bott, N. Kleinefeld, K. Neu \& K. Zickwolf (Hrsg.), Teaching Trends 2018. Die Präsenzhochschule und die digitale Transformation. Digitale Medien in der Hochschullehre, 7 (S. 30-35). Münster: Waxmann. https://nbn-resolving.org/urn:nbn:de:0111-pedocs-179180. Zugegriffen: 22. Juni 2020.

Deterding, S., Dixon, D., Khaled, R., \& Nacke, L. (2011). From game design elements to gamefulness. In A. Lugmayr, H. Franssila, C. Safran \& I. Hammouda (Hrsg.), Proceedings of the 15th international academic mindtrek conference envisioning future media environments (S. 9). New York: ACM.

Dicheva, D., Dichev, C., Agre, G., \& Angelova, G. (2015). Gamification in education: A systematic mapping study. Educational Technology \& Society, 18(3): 75-88. https://www. ifets.info/. Zugegriffen: 22. Juni 2020.

Hoyer, T., \& Mundt, F. (2017). Personalisiertes Studieren, reflektiertes Lernen. Eine Analyse des Studierverhaltens in digital gestützter Lehre. Erziehungswissenschaft, 28(55), 59-70. https://doi.org/10.3224/ezw.v28i2.7.

Kaeding, N., \& Scholz, L. (2012). Der Einsatz von Wikis als ein Instrument für Forschung und Lehre. In G. Csanyi, F. Reichl \& A. Steiner (Hrsg.), Digitale Medien - Werkzeuge für exzellente Forschung und Lehre (S. 176-186). Münster: Waxmann. https://nbn-resolving. org/urn:nbn:de:0111-opus-83182. Zugegriffen: 22. Juni 2020.

Kesselheim, W., \& Lindemann, K. (2010). Gemeinsam forschen lernen mit digitalen Medien: Das Projekt „gi - Gesprächsanalyse interaktiv“. In S. Mandel, M. Rutishauser \& E. Seiler Schiedt (Hrsg.), Digitale Medien für Lehre und Forschung. Medien in der Wissenschaft, 55 (S. 106-117). Münster: Waxmann. https://nbn-resolving.org/urn:nbn:de:0111-pedocs173230. Zugegriffen: 22. Juni 2020. 
Kultusministerkonferenz (KMK). (2019). Empfehlungen zur Digitalisierung in der Hochschullehre. https://www.kmk.org/fileadmin/Dateien/pdf/PresseUndAktuelles/2019/ BS_190314_Empfehlungen_Digitalisierung_Hochschullehre.pdf. Zugegriffen: 28. Apr. 2020.

Lee, J. Y., \& Jang, P. S. (2017). Study on research trends in airline industry using keyword network analysis: Focused on the journal articles in scopus. Journal of the Korea Convergence Society, 8(5), 169-178.

Mayrberger, K. (2008). Fachkulturen als Herausforderung für E-Learning 2.0. In S. Zauchner, P. Baumgartner, E. Blaschitz \& A. Weissenbäck (Hrsg.), Offener Bildungsraum Hochschule. Freiheiten und Notwendigkeiten (S. 157-168). Münster: Waxmann.

Pappano, L. (2. November 2012). The year of the MOOC. The New York Times.

Rodrigues, V. (2013). How to write an effective title and abstract and choose appropriate keywords. Editage insights. https://www.editage.com/insights/how-to-write-an-effectivetitle-and-abstract-and-choose-appropriate-keywords. Zugegriffen: 22. Juni 2020.

Schünemann, I., \& Budde, J. (2018). Hochschulstrategien für die Lehre im digitalen Zeitalter: Keine Strategie wie jede andere! (Arbeitspapier, 38). Berlin: Hochschulforum Digitalisierung. https://doi.org/10.5281/zenodo.2592258.

Statistisches Bundesamt. (2018). Fächergruppen, Studienbereiche und Studienfächer. Fachserie 11, Reihe 4.1, WS 2018/2019, Berlin: Destatis. https://www.destatis.de/DE/Methoden/ Klassifikationen/Bildung/studenten-pruefungsstatistik.pdf;jsessionid=093852E8A415 F6DCE434E8E3ECA6D00B.internet8741?_blob=publicationFile. Zugegriffen: 22. Juni 2020.

Open Access Dieses Kapitel wird unter der Creative Commons Namensnennung 4.0 International Lizenz (http://creativecommons.org/licenses/by/4.0/deed.de) veröffentlicht, welche die Nutzung, Vervielfältigung, Bearbeitung, Verbreitung und Wiedergabe in jeglichem Medium und Format erlaubt, sofern Sie den/die ursprünglichen Autor(en) und die Quelle ordnungsgemäß nennen, einen Link zur Creative Commons Lizenz beifügen und angeben, ob Änderungen vorgenommen wurden.

Die in diesem Kapitel enthaltenen Bilder und sonstiges Drittmaterial unterliegen ebenfalls der genannten Creative Commons Lizenz, sofern sich aus der Abbildungslegende nichts anderes ergibt. Sofern das betreffende Material nicht unter der genannten Creative Commons Lizenz steht und die betreffende Handlung nicht nach gesetzlichen Vorschriften erlaubt ist, ist für die oben aufgeführten Weiterverwendungen des Materials die Einwilligung des jeweiligen Rechteinhabers einzuholen.

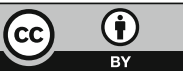

